\title{
Características dos pacientes atendidos pela primeira vez no Setor de Glaucoma da Universidade Federal de São Paulo - UNIFESP
}

\author{
Characteristics presented by patients on their first appointment at the Glaucoma \\ Sector of Universidade FederaldeSão Paulo-UNIFESP
}

\author{
Aldery de Oliveira ${ }^{1}$ \\ Augusto Paranhos Júnior ${ }^{2}$ \\ João Antônio Prata Júnior ${ }^{3}$
}

\footnotetext{
Estagiário do setor de Glaucoma da Universidade Federal de São Paulo - UNIFESP.

Doutor, chefe do setor de Glaucoma da Universidade Federal de São Paulo - UNIFESP.

${ }^{3}$ Livre-docente do Departamento de Oftalmologia, setor de Glaucoma da Universidade Federal de São Paulo UNIFESP.

Endereço para correspondência: Aldery de Oliveira Rua Diogo Farias, 650/93 - São Paulo (SP) CEP 04025-012 - E-mail: aldery@globo.com

Recebido para análise em 26.09.2002

Versão revisada recebida em 27.03.2003

Aprovação em 07.08.2003
}

\begin{tabular}{|l|}
\hline RESUMO \\
\hline Objetivo: Avaliar as características clínicas dos pacientes portadores de \\
glaucoma em um serviço universitário dereferência. Métodos: Realizou-se \\
um estudo retrospectivo e descritivo de 822 prontuários de pacientes \\
atendidos pelaprimeira vez no Setor de Glaucoma da Universidade Federal \\
de São Paulo-UNIFESP, no período de janeiro de 2000 a janeiro de 2001. \\
Os dados obtidos de cada paciente foram oriundos de uma avaliação dos \\
prontuários destes pacientes. Avaliaram-se alguns dados sociodemográ- \\
ficos e características clínicas dos pacientes glaucomatosos deste setor. \\
Resultados: A amostra era composta, em sua maioria, por pacientes \\
idosos, brancos, femininos, casados e aposentados ou do lar, com cerca \\
de 89,65\% procedentes do estado de São Paulo. Encontrou-se neste \\
estudo, 56,44\% dos olhos avaliados com acuidade visual menor que 1,0 \\
logMAR. Os casos com acuidade visual maior ou igual a 1,0 logMAR \\
corresponderam a 41,9\% dos olhos. A escavação do disco óptico já se \\
encontrava avançada (maior ou igual a 0,8) em 691 (42,03\%) olhos, sendo \\
que em 324(19,7\%) existia uma escavação total do nervo óptico. Conclu- \\
são: Observou-se que o número de pacientes em estado avançado da \\
doença era considerável, e que 41,90\% dos pacientes atendidos pela \\
primeira vez apresentavam cegueira em pelo menos um dos olhos e que \\
42,03\% apresentavam escavações do disco óptico maiores ou iguais que \\
0,8, sugerindo casos avançados de glaucoma. \\
\hline
\end{tabular}

Descritores: Glaucoma/diagnóstico; Cegueira/prevenção \& controle; Cegueira/epidemiologia

\section{INTRODUÇÃO}

O glaucoma constitui um problema de saúde pública tendo em vista a alta freqüência de incapacidade visual que acarreta ${ }^{(1-3)}$. A Organização Mundial de Saúde (OMS) sugere que $80 \%$ da cegueira encontrada nos países em desenvolvimento pode ser prevenida ou curada ${ }^{(4)}$. Segundo dados estatísticos da OMS, o glaucoma responde por cerca de 5,1 milhões de pessoas cegas ou 13,0\% da cegueira global ${ }^{(5)}$. Nos Estados Unidos, o glaucoma representa a segunda maior causa de cegueira na população geral $(11,1 \%)$ e a principal causa de cegueira na população negra ${ }^{(6-8)}$. No Reino Unido, estima-se que $0,2 \%$ da população apresenta cegueira bilateral e que o glaucoma seja responsável por 12,0\% destes casos, sendo a terceira maior causa $^{(9)}$. Um estudo realizado na Austrália, estimando a prevalência de glaucoma em 2000 e 2030, refere que haverá o dobro de pacientes com glaucoma em um período de 30 anos $^{(10)}$. 
Dentre os vários fatores envolvidos na prevenção da cegueira associada ao glaucoma, ressalta-se a detecção precoce da doença. O risco de cegueira por glaucoma está relacionado ao diagnóstico tardio da doença, quando o paciente já apresenta perda substancial de campo visual. Vários trabalhos mostram que é grande o número de pacientes que se apresentam cegos por glaucoma já na primeira consulta oftalmológica ${ }^{(1,11)}$.

Nos últimos anos, apesar da propedêutica do glaucoma ter sido acrescida de novas tecnologias, como a topografia de papila, a perimetria de freqüência dupla e de vários outros aparelhos, sob o ponto de vista de saúde pública, a principal estratégia para a detecção de glaucoma é através do exame oftalmológico de rotina. No Brasil, devido a fatores como a falta de conhecimento sobre a doença, acesso ao atendimento primário, geralmente, o diagnóstico de glaucoma é tardio, sendo o tratamento iniciado somente após o estabelecimento de profundas alterações ${ }^{(12-14)}$.

Apesar das dificuldades enfrentadas para a obtenção de dados nacionais precisos, o Conselho Brasileiro de Oftalmologia (CBO) estima que, aproximadamente 500 mil brasileiros maiores de 40 anos são portadores de glaucoma. Vinte por cento dos pacientes diagnosticados em campanhas de identificação de glaucoma não procuram assistência médica, 7,0\% das prescrições medicas não são seguidas e 40,0\% dos pacientes glaucomatosos demonstram não ter intenção de seguir o tratamento ${ }^{(15)}$. Esses números exemplificam claramente a realidade nacional, da presença de freqüências elevadas de diagnóstico tardio e baixa adesão ao tratamento ${ }^{(1)}$. Esses fatores associam-se diretamente a uma pior evolução da doença, fazendo com que sejam encontrados na população uma freqüência considerável de casos avançados que passam a requerer maior atenção especializada ${ }^{(3,16)}$. Dessa forma, casos que normalmente poderiam ser conduzidos pelo oftalmologista geral são direcionados para serviços especializados em glaucoma. A escassez destes serviços na rede pública acarreta maior demanda, agravando ainda mais a situação, tendo em vista a impossibilidade de absorver todos os $\operatorname{casos}^{(2)}$.

Assim sendo, o objetivo deste estudo é determinar as características de pacientes que procuram o Setor de Glaucoma da Universidade Federal de São Paulo.

\section{MÉTODOS}

A população estudada ( $\mathrm{N}=822)$ foi constituída por todos os pacientes atendidos pela primeira vez no Setor de Glaucoma da Escola Paulista de Medicina ou oriundos de outros serviços de oftalmologia seja do estado de São Paulo ou não, durante o período do estudo (janeiro de 2000 a janeiro de 2001).

Os dados coletados nos prontuários foram armazenados e analisados através de um estudo retrospectivo. Foram realizadas análises descritivas dos dados sócio-demográficos (idade, sexo, raça, estado civil, profissão, procedência), da história familiar de glaucoma, da acuidade visual, de cirurgias prévias, da escavação disco óptico, pressão intra-ocular, do diag- nóstico, do número de colírios em uso e tratamento instituído: clínico ou cirúrgico.

Foi analisada a distribuição por sexo entre os pacientes. Em relação à raça, os pacientes foram divididos em brancos, negros, pardos e amarelos, assim como foram divididos em grupos de casados, solteiros, separados e viúvos, quando analisado o estado civil. Em ambos os critérios, raça e estado civil, descreveu-se a representação percentual de cada grupo em relação a todos os prontuários avaliados.

A avaliação da melhor acuidade visual foi realizada através do "E" de Snelen, com valores da acuidade visual (AV) em escala decimal. Estes valores foram convertidos para escala logMAR. Foi determinado o número de olhos com acuidade visual maior ou igual a 1,0 em escala logMAR, ou seja, olhos cegos segundo a Organização Mundial de Saúde, atendidos em primeira consulta. Estes dados foram comparados tanto com a história familiar positiva ou negativa para glaucoma, como com sua manifestação entre os sexos masculino e feminino. Além disso, foi observado o número de pacientes onde a acuidade visual do melhor olho é maior ou igual a 1,0 na escala logMAR (ambos os olhos cegos).

Dados sobre a presença ou não de glaucoma na história familiar, foram computados e comparados aos níveis pressóricos preestabelecidos, independente do paciente estar ou não em tratamento clínico. A pressão intra-ocular, Po, foi dividida nos seguintes níveis, a saber: menor ou igual a $21 \mathrm{mmHg}$, de $22 \mathrm{mmHg}$ a $25 \mathrm{mmHg}$, entre 26 e $29 \mathrm{mmHg}$ e maior ou igual a $30 \mathrm{mmHg}$. O tonômetro de Goldmann acoplado à lâmpada de fenda modelo Haag Streit foi utilizado para aferir a pressão intra-ocular.

O disco óptico foi avaliado, em sua relação decimal escavação/disco, pela biomicroscopia de pólo posterior com auxílio de lentes de $78 \mathrm{D}$, sendo considerado o valor decimal da medida vertical. Com isso observou-se o número de nervos ópticos que já estavam comprometidos com uma escavação papilar maior ou igual a 0,8 .

O número de colírios em uso, o mais usado e a associação mais freqüente, foram analisados.

$\mathrm{Na}$ análise descritiva foram feitas tabelas de contingência e gráficos de setor para as variáveis categóricas e medidas resumo e gráficos do tipo box-plot para as variáveis numéricas. $\mathrm{Na}$ análise inferencial foram feitos testes de associação (quiquadrado de Pearson), para as variáveis categóricas, e test-t de Student para comparar as médias das variáveis numéricas.

\section{RESULTADOS}

Foram analisados 822 prontuários durante o período estabelecido. A idade média foi de 61,7 $\pm 16,0$ ( 8 a 93 anos). Cerca de $89,0 \%$ destes pacientes estavam entre as faixas etárias de 40 a 59 e acima de 60 anos, sendo que $71,0 \%$ deles estavam acima de 60 anos.

Encontraram-se no estudo 381 (46,35\%) pacientes do sexo masculino e $441(53,64 \%)$ do sexo feminino. 
Quanto à raça, 400 (48,7\%) eram brancos, 164 (19,9\%) eram negros, 156 (18,9\%) eram pardos, 13 (1,6\%) eram amarelos e, em 89 (10,8\%) casos, não havia informação.

Em relação à profissão dos pacientes estudados, foram descritas 76 profissões diferentes, sendo as seis mais citadas: 21 (2,55\%) eram pedreiros, 18 (2,18\%) eram estudantes, 18 (2,18\%) motoristas profissionais, 17 (2,1\%) agricultores, 201 (24,45\%) do lar, 248 (30,1\%) aposentados. Em 122 (14,8\%) casos, não foi possível determinar.

Na análise do estado civil, 448 (54,5\%) eram pacientes casados, 123 (14,9\%) solteiros, 41 (5,0\%) separados, 168 (20,4\%) viúvos e em 42 (5,1\%) casos, o estado civil não foi descrito.

Cerca de 737 (89,65\%) pacientes eram provenientes do estado de São Paulo, embora houvesse pacientes de 14 estados da federação e de um país vizinho, o Paraguai. Os estados da Bahia e de Minas Gerais, com 3,4\% dos pacientes estudados, se destacaram.

A tabela 1 revela os dados epidemiológicos dos pacientes analisados.

A acuidade visual (AV) destes pacientes foi avaliada e

\begin{tabular}{|c|c|c|}
\hline & Pacientes $(\mathrm{N}=8$ & \\
\hline \multirow[t]{4}{*}{ Idade } & $\leq 19$ & $=22(2,70 \%)$ \\
\hline & $20-39$ & $=60(7,30 \%)$ \\
\hline & $40-59$ & $=215(26,10 \%)$ \\
\hline & $\geq 60$ & $=525 \quad(63,80 \%)$ \\
\hline \multirow[t]{2}{*}{ Sexo } & Feminino & $=441 \quad(53,64 \%)$ \\
\hline & Masculino & $=381 \quad(46,35 \%)$ \\
\hline \multirow[t]{5}{*}{ Raça } & Branca & $=400 \quad(48,70 \%)$ \\
\hline & Negra & $=164(19,90 \%)$ \\
\hline & Parda & $=156(18,90 \%)$ \\
\hline & Amarela & $=13(1,60 \%)$ \\
\hline & Não determinada & $=89(10,80 \%)$ \\
\hline \multirow[t]{5}{*}{ Estado civil } & Casado & $=448 \quad(54,50 \%)$ \\
\hline & Viúvo & $=168(20,40 \%)$ \\
\hline & Solteiro & $=123(14,90 \%)$ \\
\hline & Separado & $=41(4,90 \%)$ \\
\hline & Não determinado & $=42(5,10 \%)$ \\
\hline \multirow[t]{8}{*}{ Profissão } & Aposentado & $=248 \quad(30,10 \%)$ \\
\hline & Do lar & $=201 \quad(24,45 \%)$ \\
\hline & Pedreiro & $=21(2,55 \%)$ \\
\hline & Estudante & $=18(2,18 \%)$ \\
\hline & Motorista & $=18(2,18 \%)$ \\
\hline & Agricultor & $=17(2,00 \%)$ \\
\hline & Outra & $=177(21,50 \%)$ \\
\hline & Não determinada & $=122(14,80 \%)$ \\
\hline \multirow[t]{8}{*}{ Procedência } & São Paulo & $=737 \quad(89,60 \%)$ \\
\hline & Bahia & $=20(2,40 \%)$ \\
\hline & Minas Gerais & $=8(0,90 \%)$ \\
\hline & Pernambuco & $=6(0,70 \%)$ \\
\hline & Ceará & $=4(0,50 \%)$ \\
\hline & Goiás & $=2(0,24 \%)$ \\
\hline & Outro & $=10(1,20 \%)$ \\
\hline & Não determinada & $=35(4,30 \%)$ \\
\hline
\end{tabular}

dividida em acuidade visual maior ou igual a 1,0 e menor que 1,0 , na escala logMAR.

Dos 822 pacientes atendidos, observaram-se 928 (56,44\%) olhos com AV menor que 1,0 e 689 (41,90\%) olhos com AV maior ou igual a 1,0 logMAR. Em 1,64\% dos casos, não foi possível determinar a acuidade visual (Tabela 2).

No grupo de AV maior ou igual a 1,0 na escala $\log M A R$, foram incluídos pacientes com Conta dedos (CD), Movimentos de Mãos (MM), Percepção Luminosa (PL) e Sem Percepção Luminosa (SPL). Um total de 194 olhos tinham AV igual a CD, 115 MM, 79 AV PL, e em 159 olhos não havia PL. Em 27 olhos, devido a informações precárias, a acuidade visual não foi considerada.

A acuidade visual maior ou igual a 1,0 na escala logMAR foi relacionada com os diversos grupos etários e observaramse os seguintes resultados: 15 olhos $(0,91 \%)$ eram de pacientes abaixo de 19 anos, 39 olhos (2,4\%) entre os pacientes de 20 a 39 anos, 162 olhos $(9,8 \%)$ entre o grupo de 40 a 59 anos e 471 (28,6\%) olhos no grupo maior ou igual a 60 anos. Como se nota, o grupo tem em sua predominância pacientes idosos. Em $2(0,12)$ olhos, não foi possível determinar.

A mesma acuidade visual, maior ou igual a 1,0 na escala $\log$ MAR, foi relacionada com os sexos. Observaram-se 689 olhos com esta AV entre os dois sexos. No sexo feminino, foram encontrados 368 olhos (53,41\%) e, no sexo masculino, 321 olhos (46,58\%) com acuidade visual maior ou igual a 1,0 logMAR.

Dos 822 pacientes avaliados, 681 (82,84\%) não tinham história familiar de glaucoma. Cento e quatorze $(13,86 \%)$ relataram ter algum parente glaucomatoso; em 27 (3,28\%) prontuários não foi possível identificar. Quando esses dados foram confrontados com a acuidade visual, observaram-se 565 (34,36\%) olhos com acuidade visual maior ou igual a 1,0, em pacientes com história familiar negativa para glaucoma, e 94 $(5,71 \%)$ olhos em pacientes com história familiar positiva para glaucoma. Em 30 (1,82\%) olhos, não foi possível determinar (Tabela 3).

\begin{tabular}{|c|c|c|c|c|}
\hline & Olho direito & Olho & esquerdo & Total \\
\hline $\mathrm{AV}<1,0$ & 455 & & 473 & 928 \\
\hline $\mathrm{AV} \geq 1,0$ & 352 & & 337 & 689 \\
\hline Não determinadas & 15 & & 12 & 27 \\
\hline
\end{tabular}

\begin{tabular}{|c|c|c|c|c|}
\hline & \multicolumn{2}{|c|}{ Olho direito } & \multicolumn{2}{|c|}{ Olho esquerdo } \\
\hline HFG & Sim & Não & Sim & Não \\
\hline$A V \geq 1,0$ & 50 & 285 & 44 & 280 \\
\hline$A V<1,0$ & 62 & 384 & 68 & 391 \\
\hline $\mathrm{P}$ & \multicolumn{2}{|c|}{0,7} & \multicolumn{2}{|c|}{0,6} \\
\hline Total & \multicolumn{2}{|c|}{781} & \multicolumn{2}{|c|}{783} \\
\hline \multicolumn{5}{|c|}{$\begin{array}{l}\mathrm{HFG}=\text { História familiar de glaucoma; } \mathrm{AV}=\text { Acuidade visual; } \mathrm{P}=\text { Teste de qui- } \\
\text { quadrado }\end{array}$} \\
\hline
\end{tabular}


O teste qui-quadrado não detectou diferenças estatisticamente significantes para a avaliação de história familiar e acuidade visual tanto para olho direito, quanto para olho esquerdo.

Quanto à pressão intra-ocular encontraram-se os seguintes dados:

- Po menor ou igual a 21 mmHg: 1049 olhos (63,8\%);

- Po entre 22 e 25 mmHg: 186 olhos (11,3\%);

- Po entre 26 e 29 mmHg: 90 olhos (5,47\%);

- Po maior ou igual a $30 \mathrm{mmHg}$ : 276 olhos (16,8\%);

Em 43 (2,6\%) olhos, não foi aferida a Po devido a doenças, daí impedindo o exame proposto, a exemplo de qualquer doença que comprometesse a regularidade e homogeneidade da superfície ocular ou foram esses olhos enucleados, eviscerados ou estavam em processo involutivo.

Ao relacionarem-se os dados da Po e história familiar para glaucoma, encontraram-se os seguintes dados: história familiar positiva para glaucoma foi encontrada em $146(8,88 \%)$ olhos com Po menor ou igual a $21 \mathrm{mmHg}$, em 24 (1,45\%) olhos com Po entre $22 \mathrm{mmHg}$ e $25 \mathrm{mmHg}$, em 13 (0,8\%) olhos com Po de 26 mmHg à 30 mmHg, e em 41 (2,55\%) olhos com Po acima de $30 \mathrm{mmHg}$. História familiar negativa para glaucoma foi encontrada em 874 (53,16\%) dos olhos com Po menor ou igual a $21 \mathrm{mmHg}$, em 154 (9,36\%) olhos com Po entre $22 \mathrm{mmHg}$ e $25 \mathrm{mmHg}$, em 75 (6,26\%) olhos com Po de $26 \mathrm{mmHg}$ a $30 \mathrm{mmHg}$, e em 224 (13,6\%) olhos com Po acima de 30 mmHg. Em 93 (5,65\%) olhos, não foi possível realizar essa associação (Tabela 4).

A informação dos valores da Po entre os pacientes com e sem história familiar de glaucoma não foi estatisticamente significante pelo teste t de Student, tanto para olho direito, quanto para olho esquerdo ( $\mathrm{p} \mathrm{OD}=0,6$; $\mathrm{p} \mathrm{OE}=0,6$ ).

A relação escavação/disco maior ou igual a 0,8 em seu maior diâmetro vertical foi observada em 691 (42,0\%) olhos, sendo que em $324(19,7 \%)$ comprometimento total do disco óptico. Em 218 (13,26\%) olhos, houve dificuldade de avaliar esta relação devido à catarata total, miose intensa com sinequias posteriores, hemorragia vítrea ou descolamento de retina (Tabela 5).

O diagnóstico mais freqüente foi o de glaucoma crônico simples ou glaucoma primário de ângulo aberto (GPAA) que ocorreu 633 (38,5\%) dos olhos avaliados, seguidos por glaucoma de ângulo fechado em 148 (9,0\%) dos olhos, glaucoma neovascular em 62 (3,71\%) dos olhos e glaucoma absoluto com 38 (2,31\%) dos olhos avaliados. Os diagnósticos suspeitos e as hipertensões oculares foram excluídos.

Observou-se que 591 (71,89\%) pacientes ou 1.066 olhos já eram tratados com colírio(s) ou droga de administração oral. Em uso de monoterapia, encontraram-se 240 (29,19\%) pacientes, 451 olhos deste total em uso de medicações ou $27,43 \%$ de todos os olhos avaliados. Em uso de duas medicações 192 (23,35\%) pacientes, 345 olhos deste total ou 20,98\% de todos os olhos analisados.

Em uso de 3 ou 4 medicações foram encontrados 55 (6,69\%) pacientes, 96 olhos ou 5,83\% de todos os olhos submetidos ao estudo. O uso de colírio em associação com drogas hipotensoras sistêmicas, como os inibidores da anidrase carbônica, (acetazolamida), foi encontrado em 100 (12,16\%) pacientes, 174 olhos ou 10,58\% de todos os olhos pesquisados.

A droga tópica mais usada foi o maleato de timolol, tanto como monoterapia ou associação com um, dois ou três colírios, sendo usado em 397 (48,29\%) pacientes, 763 olhos pesquisados. Em monoterapia o maleato de timolol foi usado em $142(17,27 \%)$ pacientes, 263 olhos, o betaxolol usado em 30 (3,63\%) pacientes, 57 olhos, a brimonidina em 16 (1,94\%) pacientes, 31 olhos e maleato de timolol (Glautimol ${ }^{\circledR}$ e Timoptol ${ }^{\circledR)}$, ambos usados em 10 (1,21\%) pacientes, 20 olhos. A associação mais usada foi maleato de timolol e brimonidina, em 64 (7,78\%) pacientes, 114 olhos, seguida de maleato de timolol e dorzolamida em 38 (4,62\%) pacientes, 65 olhos.

As associações de três medicações mais usadas neste estudo foram maleato de timolol, brimonidina e dorzolamida ou maleato de timolol, dorzolamida e latanoprost, encontrados em cerca de 8 e 7 (0,97\% e 0,85\%) pacientes respectivamente, ambos usados em 13 olhos (Tabela 6).

\section{DISCUSSÃO}

Os resultados observados neste estudo confirmam que os casos atendidos pela primeira vez no Setor de Glaucoma eram de glaucomas avançados, tendo em vista a alta freqüência de acuidade visual maior ou igual a 1,0 logMAR e a preponderância de grandes escavações. Isto é concordante com vários estudos, que confirmam a importância do diagnóstico precoce na prevenção da cegueira pelo glaucoma ${ }^{(11,17-18)}$.

Entre os fatores de risco relacionados ao diagnóstico tardio da doença estão a idade avançada e o baixo nível socioeconômico $^{(19)}$. Concordando, neste estudo encontraram-se $63,86 \%$ dos pacientes com 60 anos ou mais. Em 54,6\% da amostra existiu predominância de pacientes aposentados e do lar. É pertinente justificar que o tratamento do glaucoma se mostra associado a um custo elevado em relação à renda familiar

\begin{tabular}{|c|c|c|c|c|c|c|}
\hline & $\leq 21 \mathrm{mmHg}$ & $22-25 \mathrm{mmHg}$ & $26-29 \mathrm{mmHg}$ & $\geq 30 \mathrm{mmHg}$ & & Vazias \\
\hline Sim & $146(8,88 \%)$ & $24(1,46 \%)$ & $13(0,79 \%)$ & $41(2,49 \%)$ & 4 & $(0,24 \%)$ \\
\hline Não & $874(53,16 \%)$ & $154(9,36 \%)$ & $75(4,56 \%)$ & $224(13,62 \%)$ & 35 & $(2,12 \%)$ \\
\hline Vazias & $29(1,76 \%)$ & $8(0,48 \%)$ & $2(0,12 \%)$ & $11(0,67 \%)$ & 4 & $(0,24 \%)$ \\
\hline Total & $1049(63,80 \%)$ & $186(11,31 \%)$ & $90 \quad(5,47 \%)$ & $276(16,78 \%)$ & 43 & $(2,60 \%)$ \\
\hline
\end{tabular}




\begin{tabular}{|lrr|}
\hline \multicolumn{3}{|c|}{ Tabela 5. Classificação da escavação do disco óptico } \\
\hline Escavação disco óptico & \multicolumn{1}{c|}{ Olho direito } & Olho esquerdo \\
$<0,8$ & $307(18,60 \%)$ & $296(18,00 \%)$ \\
0,8 & $84(5,10 \%)$ & $95(5,77 \%)$ \\
0,9 & $106(6,44 \%)$ & $82(4,98 \%)$ \\
Acometimento total & $158(9,61 \%)$ & $166(10,09 \%)$ \\
Impraticável & $117(7,10 \%)$ & $128(7,78 \%)$ \\
Vazias & $50(3,00 \%)$ & $55(3,30 \%)$ \\
\hline
\end{tabular}

\begin{tabular}{|lccc|}
\hline \multicolumn{4}{|c|}{ Tabela 6. Número de colírios em uso por olho e em ambos os olhos } \\
$\mathbf{N}^{\circ}$ de colírios & $\begin{array}{c}\text { Olho } \\
\text { direito }\end{array}$ & $\begin{array}{c}\text { Olho } \\
\text { esquerdo }\end{array}$ & $\begin{array}{c}\text { Ambos } \\
\text { olhos }\end{array}$ \\
1 & 227 & 224 & 211 \\
2 & 175 & 170 & 153 \\
3 & 44 & 48 & 39 \\
$1+\mathrm{S}$ & 19 & 15 & 13 \\
$2+\mathrm{S}$ & 48 & 48 & 35 \\
$3+\mathrm{S}$ & 13 & 13 & 10 \\
Total & 526 & 518 & 461 \\
& $(31,99 \%)$ & $(31,50 \%)$ & $(28,04 \%)$ \\
S= Droga sistêmica & & & \\
\hline
\end{tabular}

da população que freqüenta um serviço público ${ }^{(20-21)}$. Estes dados sugerem que pacientes de baixa renda, que destinam parte significante de sua renda ao tratamento, podem apresentar maior risco de baixa fidelidade por impossibilidade de aquisição do medicamento, resultando no avanço da doença.

Sabe-se que o esclarecimento, não importando se feito por programas educacionais periódicos ou pelo médico em seu consultório, elevaria o percentual de diagnósticos precoces da doença ${ }^{(22)}$. Muitas vezes, os baixos índices de adesão ao tratamento estão associados à falta de compreensão da doença, propiciando um avanço desta doença ${ }^{(13-14)}$.

Fatores que podem justificar a baixa AV são o grande dano no disco óptico e dificuldades de adesão ao tratamento do glaucoma $^{(1,23-24)}$. Neste estudo, 42,03\% dos olhos apresentavam escavação maior ou igual a 0,8 . Os pacientes têm dificuldades de acesso ao sistema de saúde, fazendo com que seu primeiro contato seja tardio e que a lesão glaucomatosa avance de maneira importante. Por um lado, o sistema de atendimento primário é ineficaz para realizar o diagnóstico precoce de glaucoma, pelo número insuficiente de postos de saúde, pelo número insuficiente de profissionais capazes de diagnosticar e tratar adequadamente a doença ou por falta de condições clínicas em realizar o diagnóstico. Por outro lado, centros especializados são referência para casos avançados, sendo os pacientes muitas vezes encaminhados para os serviços terciários.

Dados deste estudo, apesar de não ser estatisticamente significante, mostram que o número de olhos com $\mathrm{AV} \geq 1$ $\operatorname{logMAR}$ foi maior entre pacientes que não tinham história familiar de glaucoma (34,36\%), quando comparados ao número de olhos de pacientes com história familiar de glaucoma
(5,71\%). Esta associação sugere que os pacientes com história de glaucoma na família procuraram o oftalmologista precocemente, sendo dado a eles maior oportunidade de tratar e permanecer com melhor visão. A presença de história familiar tem sido considerada fator que evita o diagnóstico tardio do glaucoma; no entanto, estudos não encontraram associação significativa entre história familiar e apresentação tardia dos pacientes $^{(19,25)}$.

Observou-se, neste estudo, que $71,9 \%$ dos pacientes atendidos pela primeira vez no setor de glaucoma da UNIFESP/ EPM faziam uso prévio de hipotensores oculares. O uso de algum tipo de associação medicamentosa foi relatado em aproximadamente $66,5 \%$ dos olhos. Cabe observar que o alto custo do tratamento glaucomatoso é fator importante no avanço da doença e causa importante na interrupção do tratamento, estando, muitas vezes, à frente dos efeitos colaterais e da ausência de melhora da acuidade visual ${ }^{(23)}$. Em outro estudo, ficou demonstrado que o gasto anual estimado com glaucoma foi equivalente a $13,0 \%$ da renda anual média dos pacientes. Isso mostra que, mesmo em instituição pública, o custo na renda familiar de pessoas portadoras de glaucoma é considerável ${ }^{(20)}$.

Pouco pode ser feito para os pacientes com glaucoma avançado, visto que, até o momento, não há tratamento que melhore ou recupere o campo de visão perdido pela evolução da doença. A proposta de tratamento é a estabilização da doença, de modo a diminuir os níveis pressóricos intra-oculares, diminuindo a perda contínua do campo visual e estabilizando os danos já manifestos do nervo óptico.

A orientação é essencial, já que o paciente deve estar ciente que o tratamento não trará benefício em curto prazo. Não é raro observar queixas de pacientes que não percebem melhora alguma durante o tratamento. Não compreendem que o processo do tratamento visa a prevenção de danos maiores e melhora em longo prazo.

\section{CONCLUSÃO}

Os resultados do estudo indicam que os casos atendidos pela primeira vez no Setor de Glaucoma da UNIFESP/EPM tratavam-se de glaucomas avançados, sendo que 41,9\% dos pacientes já apresentavam cegueira em pelo menos um olho.

\section{ABSTRACT}

Purpose: To evaluate characteristics of glaucoma patients at our university hospital facility. Methods: This is a retrospective study that shows comprehensive clinical factors presented by patients on their first appointment at the Universidade Federal de São Paulo - UNIFESP Glaucoma Department, from January 2000 through January 2001. The data originated from a social, demographic and comprehensive analysis of our prerecorded files. Results: The group consisted of elderly people, mostly Caucasian, female, married 
and retired, or with a domestic lifestyle, about $89 \%$ from the state of São Paulo. It was observed that 928 (56.44\%) of the studied eyes presented visual acuity lower than $1.0 \log$ MAR and that 689 (41.9\%) of the studied eyes displayed visual acuity above or equal to 1.0 logMAR. The advanced opticdisk cup, above or equal to 0.8 , was found in $42.03 \%$ of the studied eyes. A significant number of patients displayed advanced pathological conditions. Conclusions: It was observed that $41.9 \%$ of the cases presented blindness in one eye from the very onset, and that $42.03 \%$ of the eyes presented advanced cup above or equal to 0.8 , suggesting advanced glaucoma.

Keywords: Glaucoma/diagnosis; Blindness/prevention \& control; Blindness/Epidemiology

\section{REFERÊNCIAS}

1. Gullo RM, Costa VP, Bernardi L, Kara-José N. Condições visuais de pacientes glaucomatosos em um hospital universitário. Arq Bras Oftalmol 1996;59:147-50.

2. Costa VP, Almeida GV, Kara-José N. Prevenção da cegueira por glaucoma. Arq Bras Oftalmol 1998;61:356-60.

3. Rodrigues AC, Silva MRBM, Schellini SA. Número de olhos cegos por glaucoma detectados em primeira consulta num hospital universitário. Arq Bras Oftalmol 1998;61:573-8.

4. Thylefors B, Negrel AD, Parajasegaram R. Epidemiologic aspects of global blindness prevention. Curr Opin Ophthalmol 1992;3:824-34.

5. Thylefors B, Negrel AD, Parajasegaram R, Dadzie KY. Global data on blindness. Bull World Health Organ 1995;73:115-21.

6. Tielsch JM, Sommer A, Katz J, Quigley H, Ezrine MS. Socioeconomic status and visual impairment among urban Americans. Arch Ophthalmol 1991;109:637-41.

7. Seddon JM. The differential burden of blindness in the United States [comment in N Engl J Med 1991;325:1412-7]. N Engl J Med 1991;325:1440-2.

8. Hiller R, Kahan HA. Blindness from glaucoma. Am J Ophthalmol 1985; 80:62-9
9. Sorsby A. The incidence and causes of blindness in England and Wales, 1963 - 68; with an appendix on services available for incipient blindness. [monograph online]. London; 1972 [cited 2003 April 2]. Available from: URL: http:www.healthlinkusa.com/bookpage/47_12.html

10. Rochtchina E, Mitchell P. Projected number of Australians with glaucoma in 2000 and 2030. Clin Experiment Ophthalmol 2000;28:146-8.

11. Grant WM, Burke JF. Why do some people go blind from glaucoma? Ophthalmology 1982;89:991-8.

12. Prata JR. A importância da aderência ao tratamento do glaucoma. Novas perspectivas no tratamento do glaucoma. In: XXXI Congresso Brasileiro de Oftalmologia; 2001 set 5-9; São Paulo. Anais. p.3-5.

13. Zimmerman TJ, Zalta AH. Facilitating patient compliance in glaucoma therapy. Surv Ophthalmol 1983;28:252-8.

14. Granstrom PA. Glaucoma patients not compliant with their drug therapy: clinical and behavioral aspects. Br J Ophthalmol 1982;66:464-70.

15. Silva FA. Consenso da Sociedade Brasileira de Glaucoma. São Paulo: BG Cultural; 2001.

16. Quigley HA. Number of people with glaucoma worldwide [comment on $\mathrm{Br} \mathrm{J}$ Ophthalmol. 1996;80:385-6]. Br J Ophthalmol 1996;80:389-93.

17. Perkins ES. Blindness from glaucoma and the economics of prevention. Trans Ophthalmol Soc UK 1978;98:293-5.

18. Miller SJK, Karsearas AG. Blind registration and glaucoma simplex. Br J Ophthalmol 1974;58:455-61.

19. Fraser S, Bunce C, Wormald R. Risk factors for late presentation in chronic glaucoma. Invest Ophthalmol Vis Sci 1999;40:2251-7.

20. Pedroso L, Carvalho Jr. ES, Paranhos JR A, Prata Jr JA, Mello PAA. Custo real do tratamento do glaucoma para o paciente. Arq Bras Oftalmol 1999; 62:677-82.

21. Silva LMG, Vasconcelos JPC, Temporini ER, Costa VP, Kara-José N. Tratamento clínico do glaucoma em um hospital universitário: custo mensal e impacto na renda familiar. Arq Bras Oftalmol 2002;65:299-303.

22. Kim S, Stewart JF, Emond MJ, Reynolds AC, Leen MM, Mills RP. The effect of brief education program on glaucoma patients. J Glaucoma 1997;6: 146-51.

23. Costa VP, Vasconcelos JP, Pelegrino M, Kara-José N. O que os pacientes sabem sobre glaucoma? Arq Bras Oftalmol 1995;58:36-41.

24. Kara-José N, Saba HC, Cartocci AA, Braga ACA, Shimoda GA, Guidi HC, et al. Conhecimentos e práticas em saúde ocular de 1000 pessoas da cidade de Campinas. Arq Bras Oftalmol 1985;48:160-4.

25. Leal BC, Medeiros FA, Oliveira BFT, Pinheiro, Susanna Jr. R, Kara-José N. Fatores associados ao conhecimento de glaucoma numa população de hospital terciário. Rev Bras Oftalmol 2001;60:556-62.

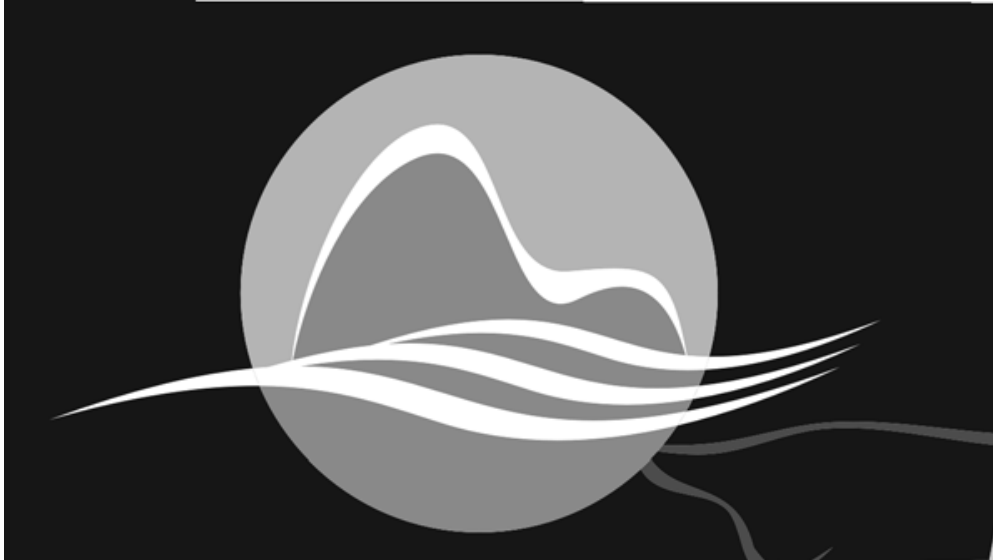

XVI CONGRESSO BRASILEIRO DE PREVENÇÃO DA CEGUEIRA E REABILITAÇÃO VISUAL

04 a 07 de setembro de 2004 Riocentro - Pavilhão 5 - Rio de Janeiro

\section{Marque em sua agenda}

04 a 07 de setembro de 2004 Riocentro - Pavilhão 5 - Rio de Janeiro

\section{Informações}

LK Ltda Secretaria Executiva/Organização:

Tel.: (21) 3878-2512

Fax: (21) 3860-6064

E-mail: evento@lk.com.br

HP: www.cboprevcegueira04.com.br

CBO

Realização:

Tel.: (11) 3266-4000

Fax: (11) 3171-0953

E-mail: eventos@cbo.com.br 